\title{
Optimization of PSF Membrane Transport Properties with the Use of Porogenic Additive
}

\author{
Supriya S. Dhume, Pallavi Mahajan-Tatpate, Yogesh J. Chendake* \\ Department of Chemical Engineering, Bharati Vidyapeeth (Deemed to be) University, \\ College of Engineering, Pune, India
}

Submitted: 06/08/2020. Revised edition: 28/10/2020 Accepted: 28/10/2019. Available online: 19/11/2020

\begin{abstract}
The porous membrane works upon sieving mechanism, where the separation and transport properties are dependent upon membrane morphology and porosity. This porosity and pore size are dependent upon membrane materials and formation parameters. Polysulfone (PSF) is one of the widely used membrane material due to its stability properties. Current work is targeted towards optimization of PSF based membranes by varying dope solution concentration and composition with the use of polyethylene glycol (PEG (MW6000)) as porogen in dope solution to enhance the transport rate and selectivity. An increase in PEG rejection with linear decrease in water flux and pore size was observed with the increase in dope solution concentration. Uniform membranes formation without any abnormal pore-size is observed from transport properties. The use of PEG as porogen leads to increased porosity due to leaching of Porogen during the phase inversion, which resulted in enhanced transport rate $(85 \%)$. Further the formed membranes maintained pore size as seen from bubble point, rejection and pore size analysis. This resulted in maintenance of selectivity. Such increased transport with high selectivity is highly essential when applicability of membranes in industrial processes like process separation and waste treatment are considered. This would lead to large industrial benefits.
\end{abstract}

Keywords: Polysulfone (PSF), Polyethylene-glycol (PEG), pore size, water flux and bubble point, transport optimization

\subsection{INTRODUCTION}

Membranes and membrane technology possess ability to provide a potential solution to the world water and energy crises [1]. Today purification of water is highly essential before its usage for potable, agricultural and animal application due to its limited availability, and contamination by industrial waste components and other harmful components [2]. A large percentage of water is used for industrial application [1]. It gets contaminated during processing and its disposal affects the quality of natural reservoirs. Such quality deterioration of water would limit its applicability for domestic and agricultural purpose [3]. Hence treatment and purification of this water is highly essential before its application.

Additionally, purification and recovery of components from process streams is one of the important parts in industries. These separation operations can affect the industrial economy to a greater extent [3]. All these factors require a techno-economical process for separation. Membrane separation and membrane technology can play a major role in the purification and 
separation processes [4]. Secondly, as membrane technology works on physical separation and recovered components can be used directly in further processing without compromising their properties [4]. For the optimum usage and applicability careful formation and property optimization of membranes is highly essential. The membrane properties are dependent upon membrane material and formation parameters.

Different types of material viz.; polymeric, inorganic and metallic are used in membrane preparation [5]. Polymeric membranes are preferable due to ease of formation and property tuning [6]. These membranes are playing a significant role in field such as water purification. They can be used for several industrial applications, such as gas separation, RO, UF, MF, catalytic reaction and tissue engineering [7]. Each application requires specific characteristics for membrane material and structure. In the case of MF and UF, NF, the pore size and the porosity of membrane determine the efficiency of separation and transport properties [5].

Formation of polymeric membrane with optimum properties is a challenging task. Though different methods have been reported for polymeric membrane formation, phase inversion is preferred method due to ease of formation and property optimization [5]. The optimizations of transport properties are feasible by variation in membrane preparation parameters [5]. It was first time introduced in 1960s by Loeb and Sourirajan [8] and involves a controlled transformation of a thermodynamically stable polymer solution from a liquid to a porous solid state by mixing with non-solvent [9]. There is always a discord between flux and selectivity during the formation of polymeric membranes by phase inversion [5]. The membranes with optimum selectivity in the form of molecular weight cut off (MWCO) and maximum flux would provide economical separation such membranes would be preferred in real life application.

The MWCO depends upon pore size of membranes. A reduction in pore size would reduce the MWCO but affect transport rate adversely. Hence there is need to optimize membrane formation properties. In case of polymeric membranes, the pore size can be optimized by varying membrane formation parameters and dope solution properties like concentration. A small pore size or lower MWCO membranes can be obtained by using high concentration dope solution.

All these membranes transport properties can be optimized by careful variation in membrane formation. Different methods have been reported for this optimization viz., use of additives, porogen, surface modification, chemical modification etc., in addition to optimization of membrane casting parameters. Porogen is an organic or inorganic material, which is added to the dope solution before formation of membrane [10]. It is selectively removed by leaching after formation of membranes [11]. This results in formation of voids or additional pores in the place of porogen molecules in membrane matrix. It leads to an increase in porosity while the pore size is controlled by size of porogen, its miscibility and interaction of leaching agent used for removal of porogen with base membrane matrix [12, 13]. A careful selection and optimization of Porogen would lead to enhancement in transport properties (flux and selectivity) of formed membranes [14].

Different polymers like polyethersulfone (PES), polysulfone (PSF), polyacrylonitrile (PAN), 
polyethylene (PE), polypropylene (PP) have been reported for preparation of membranes [15]. Among these polymers, PSF is preferred for membrane formation due to its properties. It is transparent, amorphous polymer with high strength and high heat resistance [16]. It has glass transition temperature in the range from 180 to $250{ }^{\circ} \mathrm{C}$ [17]. The polymer is hydrolytically stable and can undergo repeated cycles of steam sterilization [17]. The PSF based membranes show very low water absorption rate, low swelling and shrinking properties [18]. This makes PSF as a favorable material for membrane formation. Though PSF based membranes have multiple excellent properties, they have issues with hydrophilicity of formed membranes [19]. This leads to concentration polarization and fouling of membranes. It affects the transport properties of membranes. Hydrophilicity of the PSF based membranes can be improved significantly by using hydrophilic additives [20] and surface property modification.

The current work is targeted towards optimization of PSF based membranes transport properties using porogen concept. Use of hydrophilic polyethylene glycol (PEG) as porogen would enhance the transport rate and hydrophilicity of PSF based membranes. This would reduce the fouling issues. A careful optimization of PEG concentration and molecular weight would enhance transport rate while maintaining selectivity in terms of MWCO.

\subsection{EXPERIMENTAL}

\subsection{Materials}

Polysulfone (PSF) (synthesis grade
MW 75000) was procured from Otto Chemei Pvt. Ltd. $N, N^{\prime}$ - Dimethyl acetamide (DMAc) of synthesis grade was obtained from Loba Chemei Pvt. Ltd. Polyethylene Glycol (PEG) with molecular weight (MW) 600 (PEG 600), MW 1500 (PEG 1500), MW 6000 (PEG-6000), MW 9000 (PEG9000) and MW 20000 (PEG-20000) were obtained from High Purity Lab. Pvt. Ltd., Sisco Research Lab Pvt. Ltd. and Alfa Aesar respectively. Barium Chloride dehydrates $\left(\mathrm{BaCl}_{2} \cdot 2 \mathrm{H}_{2} \mathrm{O}\right)$, Potassium Iodide $(\mathrm{KI})$, Iodine $\left(\mathrm{I}_{2}\right)$ and Hydrochloric acid ( $\mathrm{HCl}) \quad 36 \%$ all laboratory reagent grades were purchased from Merck Specialties Pvt. Ltd., ACME Chemicals, Poona Chemical Lab, and Avra Synthesis Pvt. Ltd., respectively. Nonwoven Polyester backing of grade 3324 was obtained from Ahlstrom Hollytex.

\subsection{Solution Preparation}

Dope solution of PSF was prepared using DMAc as solvent. PSF granules were dried at $60{ }^{\circ} \mathrm{C}$ for $48 \mathrm{hr}$ under vacuum. Pre-weighed quantity of dry PSF was added to known quantity of DMAc for preparation of dope solution under constant stirring. Concentration of PSF in dope solution was varied from $21-29 \%(\mathrm{w} / \mathrm{v})$ of DMAc. The solution was stirred for $48 \mathrm{hr}$ under observation, to confirm complete dissolution of PSF in DMAc. Preweighed quantity of PEG (viz. PEG 6000, PEG 1500, PEG 600 or PEG $400)$, ranging from $2-10 \%(\mathrm{w} / \mathrm{w})$ of PSF was added to the solution while stirring.

\subsection{Membrane Casting}

Dope solutions were degassed using probe sonicator (Johnson Plastosonic, Model No. JP578L) for 2 - 5 min cycle each time to remove entrapped air bubbles. Alternative a vacuum 
degassing system with precise control on vacuum was used for the purpose. The solution was further centrifuged to remove any swollen and undissolved particles. Degassed and centrifuged solution was used for membrane preparation using casting system. A casting system with precise control on solution spread duration and surface drying time was used to avoid any irregularities and uneven surface. Nonwoven polyester backing of appropriate size was attached to the glass surface using scotch tape. Gap between backing surface and doctor knife attached to membrane caster was carefully maintained. The solution was poured on backing and spread on the surface using moving doctor's knife, while avoiding the bubble entrapment. Whole assembly containing glass and backing was transferred to water bath after predefined air-drying time 10-30 sec. The air drying time was maintained at $10 \mathrm{sec}$ due to defects and uneven skin layer formation observed visually at higher drying time. This would lead to gelation of polymeric solution and formation of membrane. The casted membrane was preserved under $4^{\circ} \mathrm{C}$ temperature, before further analysis.

\subsection{Water Flux Analysis}

Pure water flux (PWF) of prepared membrane was measured using Amicon type dead end cell with active membrane area of $13.847 \mathrm{~cm}^{2}$. Prepared membrane was mounted on the cell filled with distilled water. The stirring speed was maintained to 500 rpm. Analysis was carried out at room temperature using distilled water at the pressure of 0.4 to 2 bar. Initially $25 \mathrm{ml}$ of pure water is allowed to pass though the membranes to avoid any effect of storage media on water flux. It is followed by measuring time for collecting fix amount of volume $(2 \mathrm{ml}$ or more; minimum flow time more than $3 \mathrm{~min}$ ) so as to avoid any human error.

The flux was calculated by using Eq. 1 .

$$
\mathrm{F}=\frac{\mathrm{V}}{\mathrm{A} \times \Delta \mathrm{t}}
$$

Where, $\mathrm{V}$ is volume (L) of water transported across the membrane in time $(\Delta t, h)$ through the membrane of cross-sectional area (A).

\subsection{Analysis of Bubble Point and Pore Size Distribution}

\subsubsection{Bubble Point Analysis}

Bubble point analysis was done by using water - air $\left(\sigma=72.8 \mathrm{mN} \cdot \mathrm{m}^{-1}\right.$ at room temperature, RT), and water butanol $\left(\sigma=1.8 \mathrm{mN} \cdot \mathrm{m}^{-1}\right.$ at $\left.\mathrm{RT}\right)$ combination. A wet membrane sample was mounted in the analysis cell. It was fed with dry air or water saturated butanol. The upstream pressure was increased at regular interval, sequentially till continuous air/butanol flow rate. This is the bubble point pressure. From the bubble point, maximum pore size was calculated by using Cantors Equation [21]:

$$
r_{p_{i}}=\frac{2 \cdot \sigma \cdot \cos \theta}{P_{i}}
$$

Where, $\sigma$ is surface tension $\left(\mathrm{mN} \cdot \mathrm{m}^{-1}\right)$, $\theta$ is contact angle between water - air or water - butanol combination, and $\mathrm{P}_{\mathrm{i}}$ is applied pressure.

\subsubsection{Pore Size Distribution}

Average pore size of membranes was calculated with the help of water flux and bubble point analysis using Cantors Equation (2). The number of pores per unit area is calculated using Hagen-Poiseuille's Equation given 
below [22]:

$$
\mathrm{Ni}=\left(\mathrm{J}_{\mathrm{i}}-\frac{\mathrm{J}_{\mathrm{i}-1} \cdot \mathrm{P}_{\mathrm{i}}}{\mathrm{P}_{\mathrm{i}-1}}\right) \cdot \frac{8 \eta \cdot \mathrm{l}}{\pi \cdot \mathrm{P}_{\mathrm{i}} \cdot \mathrm{r}_{\mathrm{P}_{\mathrm{i}}}^{4}}
$$

Eq. 3

Where, Ni is the number of pores per unit area, $r_{p i}$ is the radius of the pore, $\sigma$ is the viscosity of water, 1 is pore length that is assumed to be equal to the membrane skin layer thickness in $\mu \mathrm{m}$ and $\mathrm{J}_{\mathrm{i}}$ corresponds to the flux measured at the $i^{\text {th }}$ increment where the applied pressure is $P_{i}$.

\subsection{PEG Rejection Analysis}

Molecular weight cut off (MWCO) of the formed membrane was measured using PEG rejection analysis. This analysis was performed in dead end cell. $0.1 \%$ PEG solution was prepared in water and its transport was measured. Initially $25 \mathrm{ml}$ of permeate was discarded and further material was considered for analysis. The concentration of PEG in the feed and permeate was analyzed. Concentration of PEG was measured by using UV analysis [23]. In a $0.6 \mathrm{ml}$ feed or permeate sample, $3 \mathrm{ml}$ of reagent a (1.27 $\mathrm{g} \mathrm{I}_{2}$ in $2 \%$ of KI solution) was added and mixed thoroughly. These samples were allowed to stand for 30 minutes at RT and then absorbance was measured at $535 \mathrm{~nm}$ wavelength by using double beam UV - visible spectrophotometer (Spectra scan $3000+)$ from Lab India. Percent rejection (\%) was calculated by;

$$
\% R=\left[1-\frac{C_{p}}{C_{f}}\right] \times 100
$$

Eq. 4

Where $C_{p}$ and $C_{f}$ is concentration of permeate and feed concentration respectively.

\subsection{Membrane Resistance}

The membrane resistance is calculated according to Darcy's law (Eq. 5). Membrane resistance (MR) was calculated by,

$$
M R=\frac{P}{J w \times V i s c o s i t y}
$$

Where, $\mathrm{P}$ is pressure, $\mathrm{JW}$ is Flux $\left(\mathrm{m}^{3} / \mathrm{m}^{2} . \mathrm{sec}\right)$ and viscosity of water is $8.90 * 10^{\wedge}(-4)\left(\mathrm{m}^{-1}\right)[5]$.

\subsection{Membrane Scanning Electron Microscopic (SEM) Analysis}

The membrane surface morphology (porosity and pore size) was analyzed using Nova Nano SEM NPEP303 FESEM system and TLD detector at different magnification from 20000 to 200000. The polymeric membrane samples was coated with gold and analyzed at $5 \mathrm{kV}$.

\subsection{RESULTS AND DISCUSSION}

\subsection{Effect of Dope Solution Polymer Concentration on Transport Properties}

The asymmetric porous polymeric membranes are preferably prepared by phase inversion method. It requires careful selection of polymer, solvent and non-solvent combination for formation of membrane. Though various non-solvents are possible, but water is most preferable non-solvent due to its economic benefits and large scale availability for membrane formation [5]. This limits the availability of polymers as membrane material [24]. Amongst various polymers reported PSF is selected for the current work due to its stability, mechanical strength, chemical inertness and good film forming ability 
$[14,25]$. Another key feature of the PSF membranes is that they hardly swell or shrink, which will maintain transport properties at high pressure [26, 27 and 28]. There is limitation on maximum PSF concentration in dope solution. This limits the minimum pore size of these membranes; which is majorly controlled by dope solution concentration [27]. This required optimized of membranes by choice of solvent and dope solution composition modification.

Commonly, the PSF based membranes are prepared using $N, N^{\prime}-$ dimethyl formamide (DMF) and $\mathrm{N}$ methyl pyrrolidone (NMP) as solvents $[29,30]$. They have limit on maximum solubility of PSF in solution [27]. The PSF solution in DMF as a solvent reported to be unstable at concentration above $19 \%(\mathrm{w} / \mathrm{w})$ [31, 32]. Hence DMAc was used as solvent in this work, which showed very high solubility of PSF. The PSF solutions are stable till the concentrations upto $40 \%(\mathrm{w} / \mathrm{w})$, for PSF molecular weight of $25 \mathrm{kDa}$. Such higher concentration would be useful to obtain dense surface layer. It would result in the desired selectivity properties. At the same time DMAc is highly miscible with water, commonly used as nonsolvent in gelation bath during preparation of membranes by phase inversion. This high polymer solubility and easy miscibility made DMAc a favorable choice for use as solvent for dope solution preparation in the current work.

Concentration of PSF in dope solution has strong effects on the transport properties of formed membrane. An increase in dope solution concentration tends to form the denser top layer with smaller pore size [33]. Such reduction in pore size would reduce membrane flux or permeation rate and increase rejection properties [34]. The effect of dope solution concentration in DMAc was investigated by varying the PSF (MW $75 \mathrm{kDa}$ ) concentration from 23 to $29 \%$ $(\mathrm{w} / \mathrm{v})$. Formed membranes were analyzed for water transport, bubble point and molecular weight cut off (MWCO).

\subsection{Water Flux Analysis}

Pure water flux (PWF) analysis plays an important role in defining transport properties of porous membrane. These flux and permeation properties control the application of membrane. Higher flux at low pressure is highly desirable for any particular application. It would result in higher output with low energy consumption. Hence the water flux of formed membranes was investigated at different pressures. At two bar pressure an exponential reduction in water flux with the increasing dope solution concentration was observed (Figure 1). Water flux was decreased sharply from 611 to $68 \mathrm{lmh}$ with increase in dope solution concentration from 23 to $29 \%$ $(\mathrm{w} / \mathrm{v})$. 


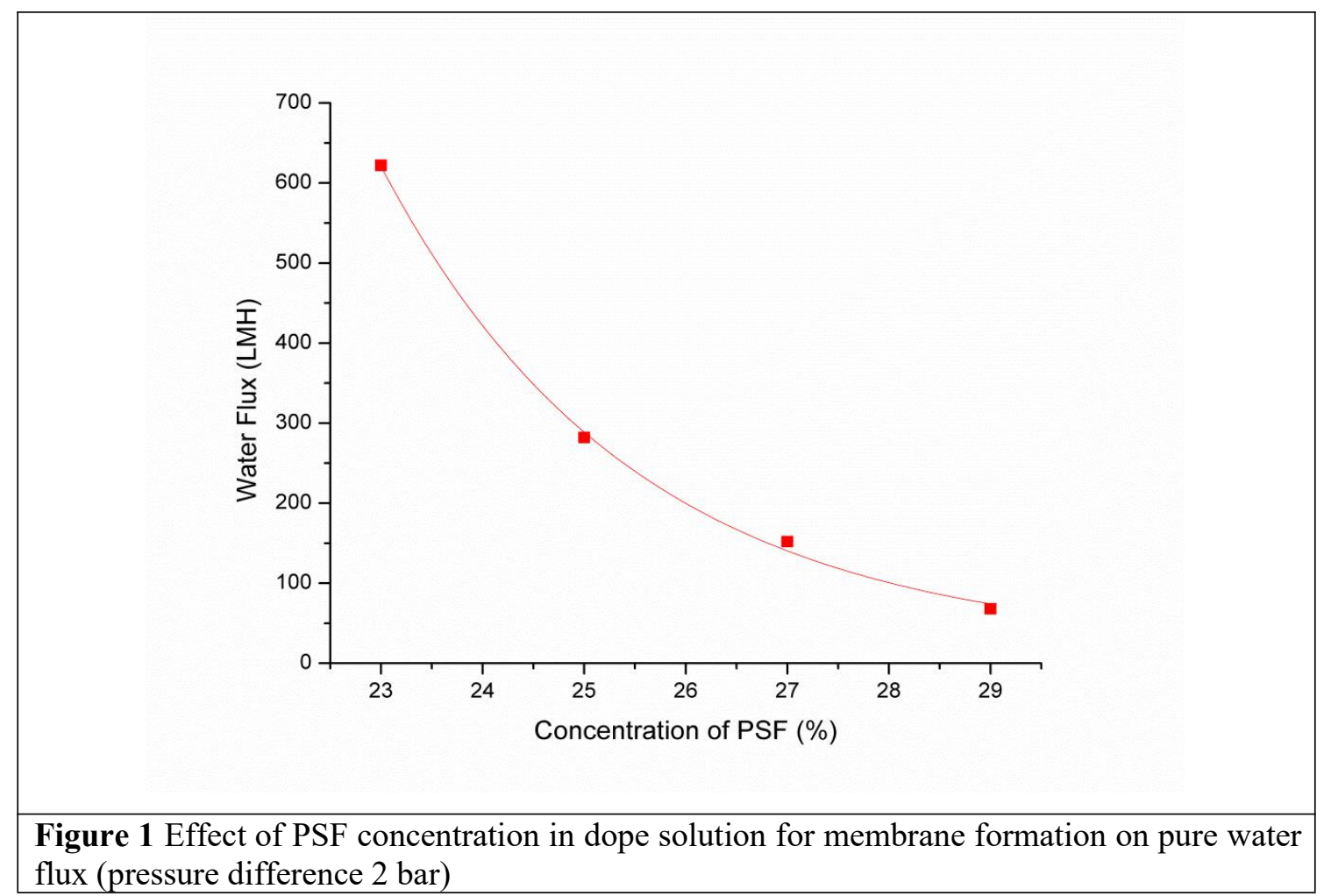

The variation in PWF has different effect on the membranes prepared with different dope solution concentration
(Table 1). The reduction in PWF with pressure was decreased with increase in the dope solution concentration.

Table 1 Effect of pressure on pure water flux for membranes with different PSF concentration in dope solution

\begin{tabular}{|l|l|l|l|l|}
\hline \multirow{2}{*}{$\begin{array}{l}\text { Driving } \\
\text { Pressure (bar) }\end{array}$} & \multicolumn{4}{|l|}{ Pure Water Flux J $\left(\mathbf{l m}^{-\mathbf{2}} \mathbf{h}^{-1}\right)$ on Concentration of PSF in Membranes } \\
\cline { 2 - 5 } & $\mathbf{2 3 \%}$ & $\mathbf{2 5 \%}$ & $\mathbf{2 7 \%}$ & $\mathbf{2 9 \%}$ \\
\hline 0.4 & 164 & 67 & 44 & 17 \\
\hline 0.8 & 291 & 131 & 69 & 31 \\
\hline 1.2 & 390 & 175 & 102 & 46 \\
\hline 1.6 & 496 & 244 & 131 & 57 \\
\hline 2 & 611 & 282 & 152 & 68 \\
\hline
\end{tabular}

This variation can be attributed to variation in membrane pore size and porosity. During the gelation, a contact of solution with non-solvent triggers the leaching of solvent. It results in polymer rich phase and leads to rearrangement in polymer solution. This would form a porous layer of polymer from polymer rich and lean areas by rearrangement and alignment. The increase in concentration of dope solution would make it polymer rich. This solution upon dipping in non-solvent bath would restrict to lower rearrangement and smaller pores.

Such reduction in pore size would result in higher resistance for PWF, thus reduction in same was observed. Similar reduction in PWF with increase in dope solution concentration is reported for 
PAN, PSF [35]. These effects of dope solution concentration on pore size and porosity can be seen strongly from bubble point, pore size and rejection analysis in Table 2.

Table 2 Variation in membrane properties with the increase in PSF concentration of dope solution

\begin{tabular}{|l|l|l|l|}
\hline $\begin{array}{l}\text { Concentration of } \\
\text { PSF (\%) }\end{array}$ & $\begin{array}{l}\text { Water Flux J } \\
\left(\mathbf{I M}^{-2} \mathbf{h}^{-\mathbf{1}}\right)\end{array}$ & $\begin{array}{l}\text { Bubble point } \\
(\mathbf{b a r})\end{array}$ & $\begin{array}{l}\text { Pore size } \mathbf{r}_{\mathbf{p}} \\
(\mathbf{n m})\end{array}$ \\
\hline 23 & 622 & 0.2 & 1404 \\
\hline 25 & 282 & 0.8 & 351 \\
\hline 27 & 152 & 2.9 & 96 \\
\hline 29 & 68 & 5.6 & 50 \\
\hline
\end{tabular}

\subsection{Bubble Point Analysis and Pore Size}

The effect of variation in dope solution concentration on membrane properties was further investigated using bubble point analysis. Bubble point is the minimum pressure required to open the largest pores of membrane to provide a continuous flow of fluid [36]. The bubble point measurement was carried out using air-water or butanol-water combination. Formed membranes showed an exponential increase in airwater bubble point (from 0.2 to 5.6 bar) with the increase in dope solution concentration from 23 to $29 \%(\mathrm{w} / \mathrm{v})$ (Table 2). Such increase in bubble point could be attributed to reduction in pore size with increase in dope solution concentration. Higher pressure would be required to displace water from the pores of membrane and get a continuous flow of air. It results in increased bubble point at higher dope solution concentration. A decrease in pore size with increase in dope solution concentration is well reported [37]. These observations of reduction in pore size at higher dope solution concentration were supported by reduction in PWF as discussed above.

Bubble point and transport analysis were further used in pore size determination. The pore radius of formed membranes was calculated using bubble point analysis, from Equation 2 [5]. As seen from Figure 2; the pore size of formed membranes was reduced from 1404 to $50 \mathrm{~mm}$ with increase in dope solution concentration from 23 to $29 \%$ $(\mathrm{w} / \mathrm{v})$. An exponential decrease in pore size is observed as opposite to the increase in bubble point in the same concentration variation. The pore size was reduced by $96 \%$, with the increase in dope solution concentration of $6 \%$ (from 23 to $29 \%$ ). This can be attributed to higher polymer or lower solvent content in dope solution. It would affect surface drying and gelation kinetics of the solution. Such a variation in gelation would result in lower pore size. Same has been supported by PWF and bubble point analysis of the formed membranes as discussed above. A decrease in pore size with increase in dope solution concentration is reported in literature [33].

A curious observation was seen in case increase in PSF concentration from 21 to $23 \%$ in dope solution. The change in bubble point was negligibly small while change in pore size was significant (Figure 2). This was attributed to abnormal pore formation at $21 \%$ dope solution concentration and hence the membranes were not used in analysis further. 


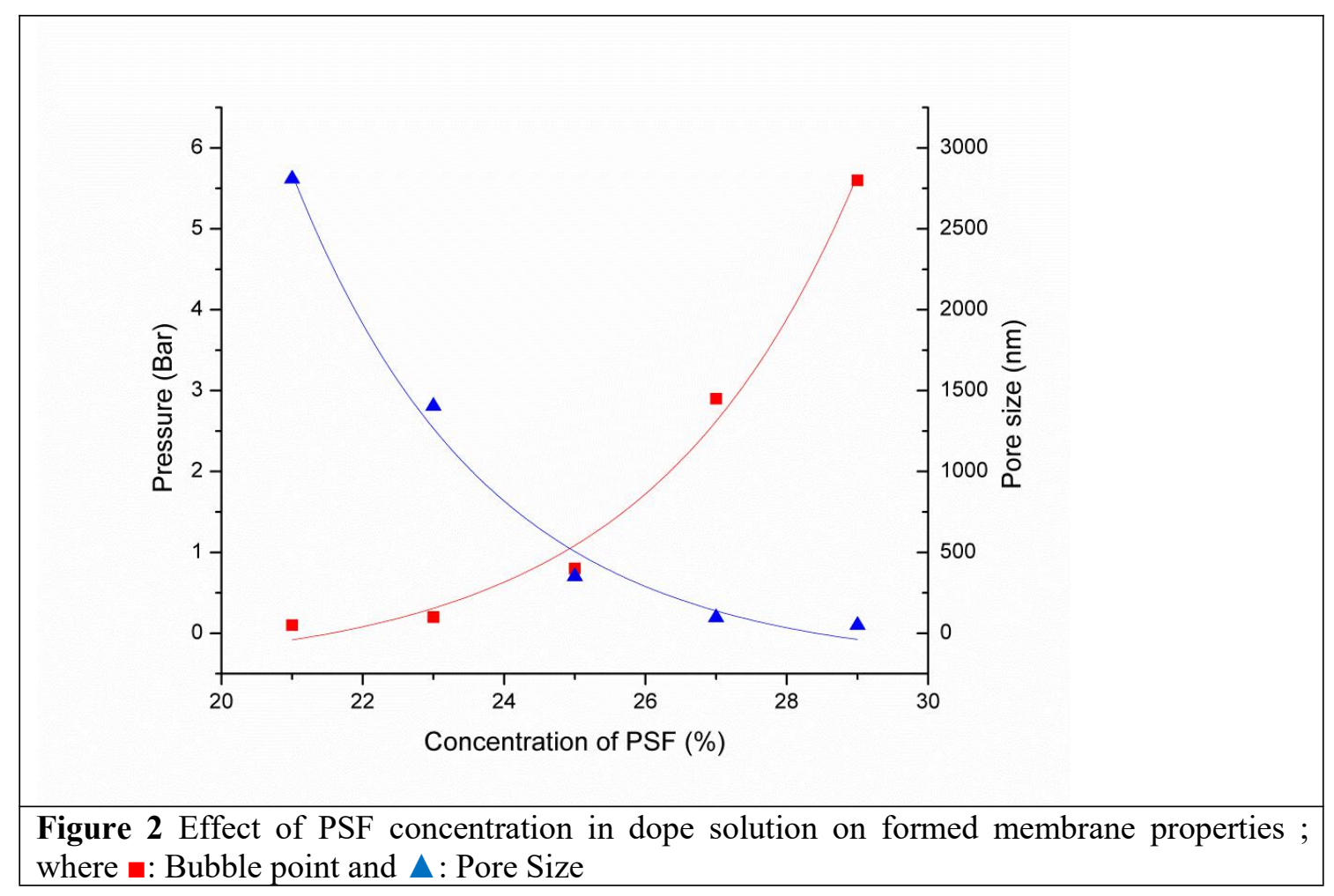

\subsection{Effect of Driving Pressure on PWF}

As described in earlier section the PSF based asymmetric membranes were prepared by phase inversion. The asymmetric membrane consists of thin skin layer with very low pore size with bottom support layer having large porous structure. Formation of skin layer with small pores controls permeation and selectivity properties, while porous layer provides mechanical support. A reduction in pore size of skin layer is observed at higher dope solution concentration. Reduction in pore size with the increase in dope solution concentration is reported [38].

Further applicability of membranes is dependent upon stability of membranes at operating conditions. One of the major factors is compaction of membranes due to collapse of pores from skin layer at operating pressure. The skin layer of asymmetric membrane can collapse under external pressure during real life application. It would affect the membrane transport properties adversely. Hence it was necessary to investigate the stability of membrane under increasing operational pressure, though stability of these membranes is expected to be higher due to high mechanical stability of PSF. It was investigated by analyzing variation in water flux with increasing pressure $(0.4$ to 2 bar), which is the general operational range of UF membranes.

A linear increase in water flux was observed with the increase in pressure from 0.4 to 2 bar in Figure 3. This can be attributed to increasing driving force responsible for transport properties. Such increase in flux with driving pressure is well reported in the literature [39]. The membrane based upon lower dope solution concentrations showed more increase in water flux with pressure as compared to membranes with higher dope solution concentration in Figure 3. This can be attributed the opening of a higher number of pores 
with an increase in pressure, which would lead to an enhanced flux. The pore sizes are less for membranes prepared from high dope solution concentration as discussed earlier. It would require high pressure and pores would open uniformly at all these pressures. Hence there would be a large resistance for water flow through the pores and a lower increase in flux with pressure was observed for the membranes formed from solutions with higher dope solution concentration. A uniform pore formation and low increase in flux for the membranes with higher dope solution concentration is reported in literature [40].

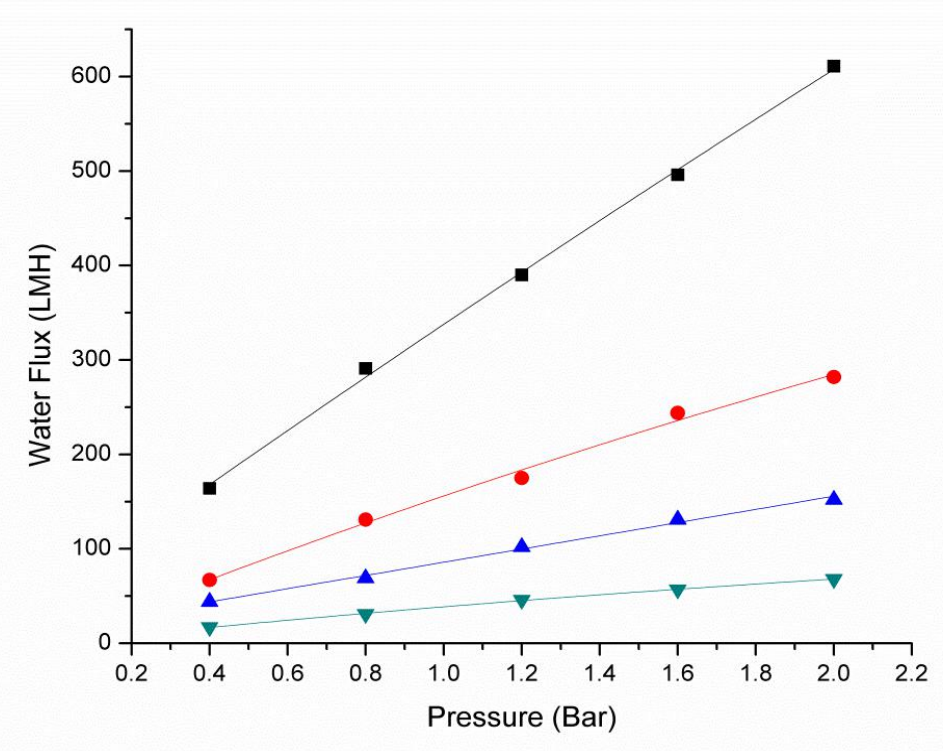

Figure 3 Variation in water flux with increase in pressure difference across membranes, where PSF concentration in dope solution $\mathbf{~} 23 \%, \bullet 25 \%, \boldsymbol{\Delta} 27 \%$ and $\nabla 29 \%$

$\begin{array}{ll}\begin{array}{l}\text { 3.5 Polyethylene Glycol (PEG) } \\ \text { Rejection Analysis }\end{array} & \begin{array}{l}\text { in rejection properties due to interaction } \\ \text { with membrane surface. Different PEG's } \\ \text { viz., PEG 20000, PEG 9000, PEG 6000 }\end{array} \\ \begin{array}{l}\text { The effect of variation in dope solution } \\ \text { with a molecular weight of 20000, 9000 } \\ \text { concentration on pore size and } \\ \text { membrane morphology was further }\end{array} & \begin{array}{l}\text { The rejection analysis was carried out at } \\ \text { analyzed by PEG rejection analysis. }\end{array} \\ \begin{array}{l}\text { PEG was selected due to their neutral trans-membrane pressure as } \\ \text { reported in Sim (1980) and results are }\end{array} \\ \text { nature. This would avoid any variation }\end{array}$

Table 3 Effect of PSF concentration in dope solution on PEG rejection properties

\begin{tabular}{|c|c|c|c|c|}
\hline \multirow{2}{*}{$\begin{array}{c}\text { Molecular } \\
\text { Weight of PEG }\end{array}$} & \multicolumn{4}{|c|}{ PEG Rejection (\%) on Concentration of PSF \% } \\
\cline { 2 - 5 } & $\mathbf{2 3 \%}$ & $\mathbf{2 5 \%}$ & $\mathbf{2 7 \%}$ & $\mathbf{2 9 \%}$ \\
\hline 6000 & 6 & 22 & 57 & 85 \\
\hline 9000 & 8 & 39 & 92 & 94 \\
\hline 20000 & 15 & 87 & 91 & 95 \\
\hline
\end{tabular}


It was observed that the rejection for PEG 6000 and PEG 9000 was negligibly low for membrane with $23 \%$ dope solution concentration. Rejection was increased from 15 to $87 \%$ for PEG 20000 with $2 \%$ increase in dope solution concentration from 23 to $25 \%$ $(\mathrm{w} / \mathrm{v})$ in Table 3. It was further increased to 91 and $95 \%$ with increase in dope solution concentration to 27 and $29 \%$ respectively. Similarly, the rejection was increased from 8 to $94 \%$ for PEG 9000 and 6 to $85 \%$ for PEG 6000 with increase in dope solution concentration from 23 to $29 \%$. This large increase in rejection could be attributed to reduction in pore size. Such reduction in pore size would increase resistance for transport of PEG. This would improve PEG rejection through formed membranes. An increase in rejection and decrease in pore size with increase in dope solution concentration is reported [37]. Though the rejection for PEG 6000 was improved significantly from 6 to $85 \%$ with increase in dope solution concentration from 23 to $29 \%(\mathrm{w} / \mathrm{v})$, but still it is lower than $90 \%$. This increase in rejection is supported by the reduced PWF and pore size, and increased bubble point as discussed in earlier section.

\subsection{Modification using PEG as Porogen}

Porosity and pore size for PSF based membranes are controlled by dope solution concentration and composition. This morphology governs the selectivity and flux of the formed membranes [41], which defines practical applicability of membranes. The membranes with high selectivity and high flux are desirable for selected separation application. This can be optimized by using suitable porogen in dope solution, which is leached after formation of membranes. Further porogen would control the viscosity and gelation properties, which would affect the membrane transport properties [42, 43]. Out of different porogens reported for PSF, polyethylene glycol with molecular weight 6000 (PEG6000) was used in current investigations.

PEG 6000 was selected due to its polymeric nature and solubility in nonsolvents water. It would change total polymer concentration in dope solution, favorably changing its viscosity towards higher sideand gelation properties. Additionally, PEG is fast soluble in water, thus it would get leached in water used for gelation. It would enhance pore density with maintaining pore size, which would help to maintain the selectivity while improving flux or transport rate.

As discussed above (Section 3.5.) membrane based on dope solution containing PSF $29 \%(\mathrm{w} / \mathrm{v})$ showed $88 \%$ rejection for PEG6000. Thus, the MWCO for these membranes is nearly equivalent to $6000 \mathrm{Da}$ or pore size of the membranes higher than size of PEG6000. Hence PEG 6000 was selected as a Porogen to maintain the pore size and increase pore density of membranes.

\subsection{Effect of PEG Concentration}

Optimization of PEG concentration in dope solution is highly essential to obtain desired transport and selectivity properties. The effect of PEG content in dope solution was investigated by analyzing its effect on water flux, bubble point and pore size of formed membranes [44, 45]. The PWF across membranes increases with increase in PEG concentration in dope solution as seen in Figure 4. It was increased by almost double (from 68 to $102 \mathrm{lmh}$ ) with the addition of $2 \%$ PEG in dope solution. While it increased three times with addition of $6 \%$ PEG in dope solution. This could be attributed to increase in porosity with use of PEG as porogenic 
additive in dope solution. The porogenic additive PEG is soluble in water. Hence, dipping of membrane in water bath during gelation would lead to progressive leaching of PEG from the formed membranes. This would result in formation of additional pores without any effect on basic membrane morphology. A rapid leaching of PEG in water without any effect on thickness and area of membranes is reported [14, 46]. Such increase in porosity would result in enhanced water flux. At the same, the rejection of PEG 6000 for PSF membranes based on $29 \%(\mathrm{w} / \mathrm{v})$ dope solution is $\sim 88 \%$. Thus size of PEG 6000 is smaller compared to the pore size of these membranes. Hence the pores generated due to leaching of PEG are small and help to maintain the selectivity as seen from pore size analysis (Figure 4).

Similar increase in porosity with addition of porogen is reported. Though, water flux of the membranes was increased by three times their pore size remained practically constant in Figure 4. The maximum variation in pore size was from 50 to $58 \mathrm{~nm}$, while bubble point was decreased from 5.6 to 4.8 bar. This would be useful while maintaining selectivity properties of membranes. Though further addition of $8 \%$ PEG in dope solution showed as increase in water flux, the bubble point was decrease considerably to 0.4 bar. It may be due to formation of uneven pore size due to interconnecting pores at this condition. Such pore size would affect selectivity adversely hence $8 \%$ and higher concentration PEG was not useful further application.

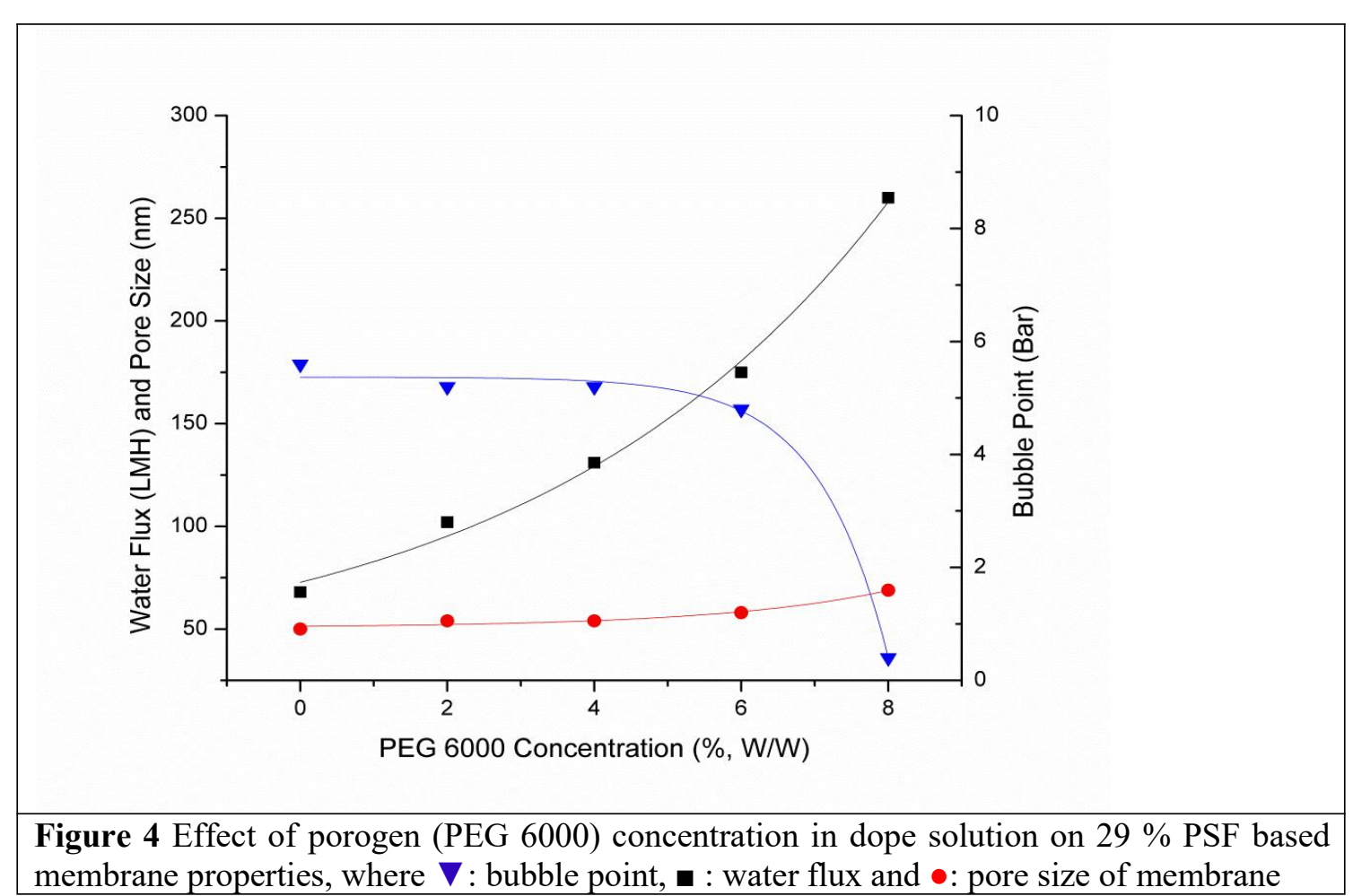

\subsection{Membrane Resistance and Transport Property Analysis}

It can be seen from Figure 5, the resistance of membrane increases exponentially with increase in PSF concentration in dope solution. This can be attributed to decrease in pore size with increase in dope solution concentration (Figure 5). This supports 
classical theory of sieving mechanism [5], where transport properties and flow are dependent on pore size of membranes. This increased resistance would reduce PWF through the membrane as seen in Figure 5.

Further, a decrease in membrane resistance and increase in PWF, while maintaining pore size was seen with the addition of PEG 6000 in dope solution.
This can be attributed to the effect of PEG 6000 as porogen during membrane formation. The porogens would be leached in water it would lead to an increase in porosity and decrease in resistance (Figure 5). This would results in higher transport rate as per the sieving mechanism, due to presence of higher number of pores.

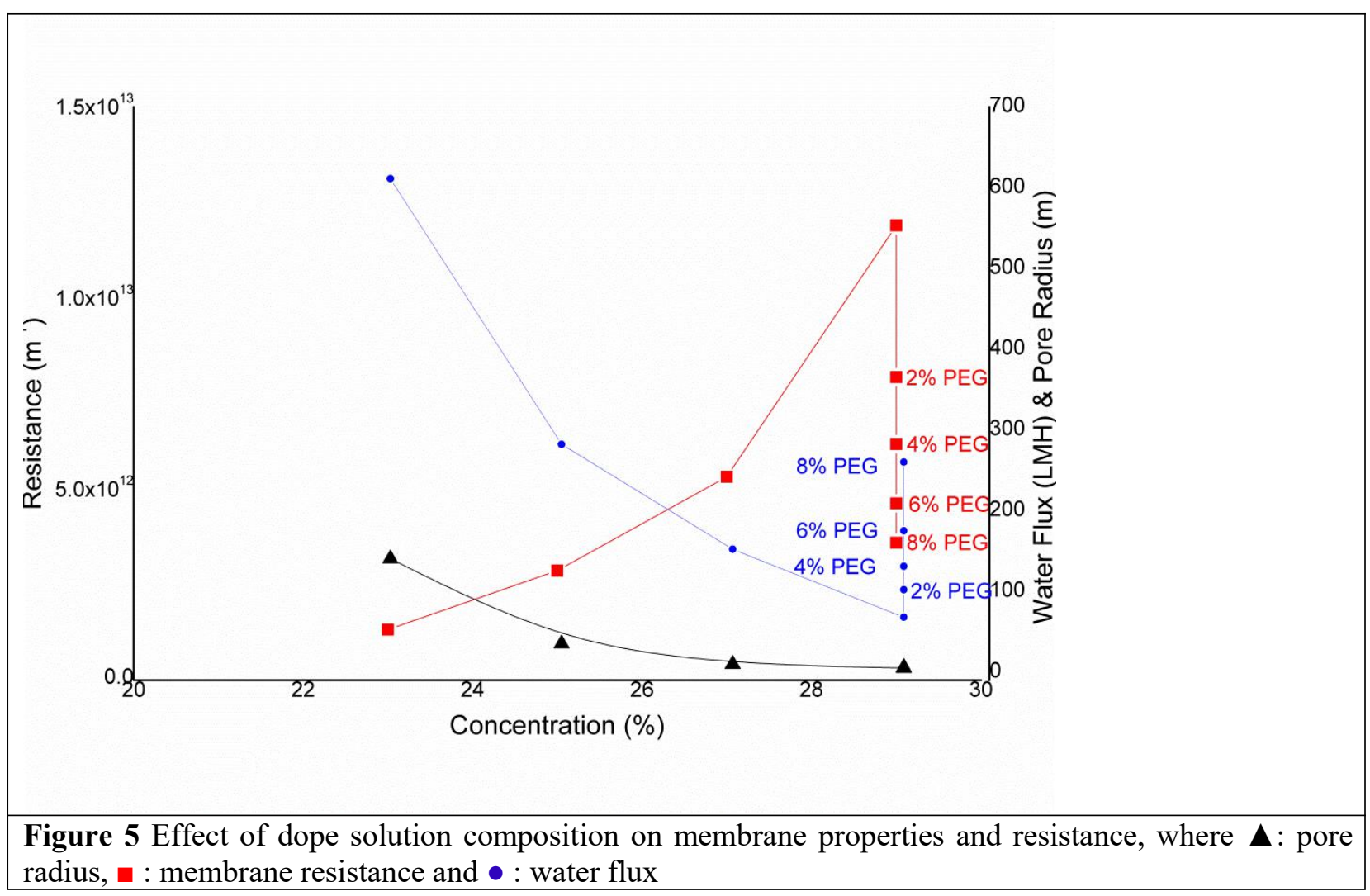

\subsection{Scanning Electron Microscopy (SEM) Analysis}

The surface morphology for membranes was further analyzed using SEM to confirm the effect of porogen on pore structure, distribution and density. Surface SEM images of $29 \%$ PSF based membranes without and with PEG (Figure 6) showed uniform surface without any abnormality. The pores were distributed evenly without any abnormal pore sizes. Both membranes showed same pore size in the range of $12-18 \mathrm{~nm}$, while increase in porosity was observed for the membrane with PEG as porogen. Such increase porosity while maintaining pore size would result in higher flux while maintaining the selectivity as seen earlier (Section 3.1). This would provide the benefits of efficient separation and with enhanced transport properties, reduction in energy requirements, and subsequent economic benefits during applications in industrial processes. 


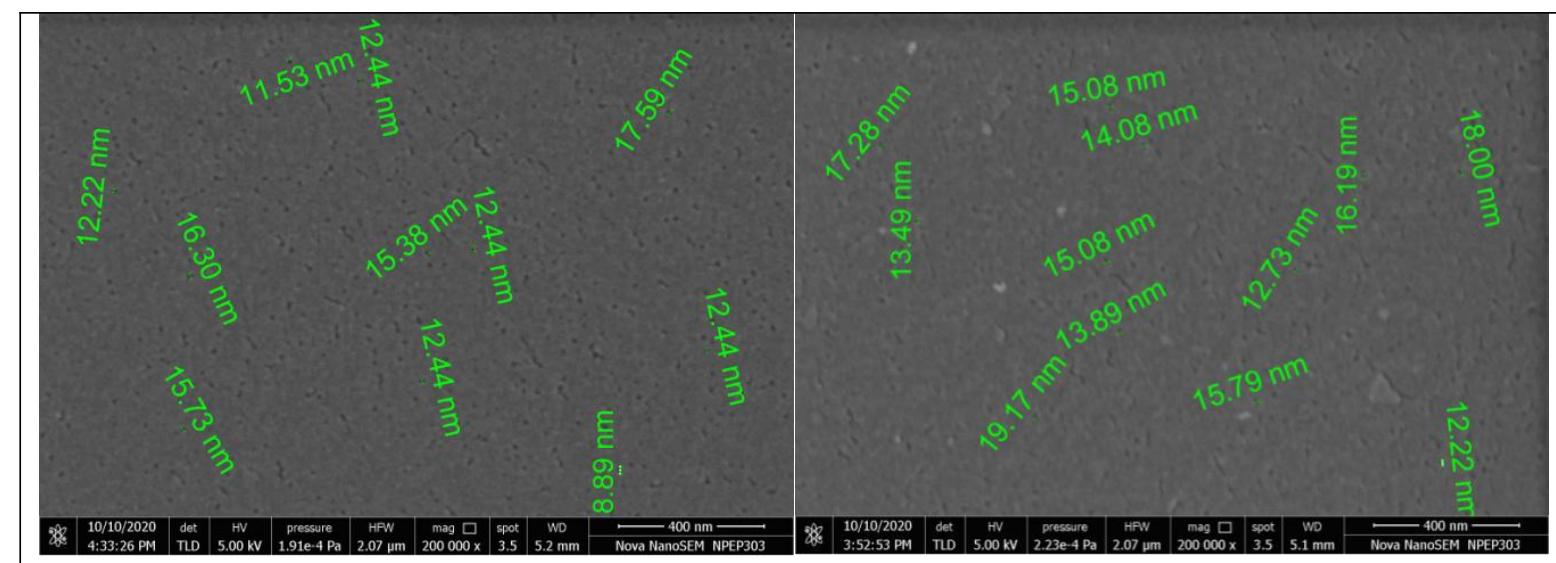

(b)

Figure 6 Effect of presence of porogen (PEG 6000) in dope solution on $29 \%$ PSF based membranes, where SEM image a: without PEG, b: with PEG $6 \mathrm{kDa}$

\subsection{CONCLUSION}

PSF is one of the extensively used polymers for preparation of UF membranes by phase inversion. These membranes have got numerous application due to stability properties of PSF. Formation of phase inversion membranes of PSF has certain limitation towards solvent selection and stability of solution at higher concentration. This limits the morphology and transport properties of PSF based membranes. This work showed use of DMAc as solvent leads to stable solution formation at higher concentration. The PSF solubility was improved to $29 \%$ $(w / v)$ of DMAc. Such increase in dope solution concentration resulted in reduction in pore size from 1404 to 50 $\mathrm{nm}$; water flux was reduced to $25 \%$ (from 17 to $68 \mathrm{lmh}$ ). Such reduction in flux restricts use of membrane at industrial level. It is always desired to prepare the membranes with low pore size and high flux. Hence PEG was used as Porogen to improve the transport properties. Use of PEG with $6 \mathrm{kDa}$ molecular weight showed 3.8 times increase in PWF (from 68 to $260 \mathrm{lmh}$ ) while maintaining the pore size and bubble point. Such increase in transport properties while maintaining the selectivity would be highly important in industrial scenario in terms of reduction in energy requirements, efficient separation and transport properties, and subsequent economic benefits. These benefits would enhance applicability of resulted membranes to a multitude level making the overall separation process technologically and energy efficient, economic, and industrially attractive.

\section{ACKNOWLEDGEMENT}

We thank Bharati Vidyapeeth (Deemed to be) University, College of Engineering, Pune for providing us the useful resources. We also thank for the financial support from DST- NanoMission (Sanction No. SR/MN/NT/1029/2015). We would like to take the opportunity to thank our family members for their understanding, as we have taken away the valuable time of togetherness to accomplish this work.

\section{REFERENCES}

[1] Abdulaziz Alammar, Sang-Hee Park, Craig J. Williams, Brian Derby, Gyorgy Szekely. 2020. Oil-in-water Separation with 
Graphene-based Nanocomposite Membranes for Produced Water Treatment. J. Member. Sci. 603: 1-11.

[2] Maria Fernanda Jaramillo and Ines Restrepo. 2017. Wastewater Reuse in Agriculture: A Review about Its Limitations and Benefits. Sustainability. 9(10): 119.

[3] Pawel Krzeminski, Edward Feys, Marc Angles d'Auriac, Aina Charlotte Wennberg, Muhammad Umar, Carsten Ulrich Schwermer, Wolfgang Uhl. 2020. Combined Membrane Filtration and $265 \mathrm{~nm}$ UV Irradiation for Effective Removal of Cell Free Antibiotic Resistance Genes from Feed Water and Concentrate. $J$. Member. Sci. 598: 1-11.

[4] Esra Eray, Vittorio Boffa, Mads K. Jorgensen, Giuliana Magnacca, Victor M. Candelario. 2020. Enhanced Fabrication of Silicon Carbide Membranes for Wastewater Treatment: from Laboratory to Industrial Scale. $J$. Member. Sci. 606: 1-11.

[5] M. Mulder. 1996. Basic Principles of Membrane Technology. 2nd ed. Netherlands.

[6] Tabassum Malik, Humaira Razzaq, Shumaila Razzaque, Hifza Nawaz, Asima Siddiqa, Mohammad Siddiq, Sara Qaisar. 2019. Design and Synthesis of Polymeric Membranes Using Water Soluble Pore Formers: An Overview. Polym. Bull. 76: 4879-4901.

[7] Bradley Ladewig, Muayad Nadhim Zemam, Al-Shaeli. 2017. Fundamentals of Membrane Bioreactors. 1st ed. Singapore

[8] D. Da Silva Biron, V. Dos Santos, M. Zeni. 2018. Ceramic Membranes Applied in Separation Processes. 1st ed. Germany. 7-29.
[9] I. M. Wienk, R. M Boom, M. A. M. Beerlage, A. M. W. Bulte, C. A. Smolders, H. Strathmann. 1996. Recent Advances in the Formation of Phase Inversion Membranes Made from Amorphous or Semi-crystalline Polymers. J. Membr. Sci. 113: 361-371.

[10] Sabri Alyarnezhad, Tiziana Marino, Jalal Basiri Parsa, Francesco Galiano, Claudia Ursino, Hermenegildo Garcìa, Marta Puche and Alberto Figoli. 2020. Polyvinylidene FluorideGraphene Oxide Membranes for Dye Removal under Visible Light Irradiation. Polym. 12(1509): 1-19.

[11] K. C. Khulbe, T. Matsuura. 2018. Removal of Heavy Metals and Pollutants by Membrane Adsorption Techniques. Appl. Water Sci. 8(19): 1-30.

[12] H. M. Aydin, A. J. El Haj, E. Piskin and Y. Yang. 2009. Improving Pore Interconnectivity in Polymeric Scaffolds for Tissue Engineering. J. Tissue Eng. Regen Med. 3: 470-476.

[13] T. D. Kusworo, Budiyono, J. Supriyadi, D. C. Hakika. 2014. Enhanced Separation Performance of Cellulose Acetate Membrane for Brackish Water Separation Using Modification of Additives and Thermal Annealing. Inter. $J$. Waste Resour. 4(1): 1-9.

[14] Akshay D. Khade, Yogesh J. Chendake. 2017. Improvement in Polysulfone (PSF) Film Properties by Porogen Concept. Int. J. Res. Appl. Sci. Eng. Technol. 5(6): 2303-2309.

[15] Muhammad Zahid, Anum Rashid, Saba Akram, Zulfiqar Ahmad Rehanand Wasif Razzaq. 2018. A Comprehensive Review on Polymeric Nano-composite 
Membranes for Water Treatment. J. Membr. Sci. Technol. 8(1): 120.

[16] Kerstin Muller, Elodie Bugnicourt, Marcos Latorre, Maria Jorda, Yolanda Echegoyen Sanz, Jose M. Lagaron, Oliver Miesbauer, Alvise Bianchin, Steve Hankin, Uwe Bolz, German Perez, Marius Jesdinszki, Martina Lindner, Zuzana Scheuerer, Sara Castello and Markus Schmid. 2017. Review on the Processing and Properties of Polymer Nanocomposite and Nanocoatings and Their Applications in the Packing, automotive and Solar Energy Fields. Nanomater. 7(74): 1-47.

[17] Meriem Nadour, Fatima Boukraa, Adel Ouradi and Ahmed Benaboura. 2017. Effects of Methylcellulose on the Properties and Morphology of Polysulfone Membranes Prepared by Phase Inversion. Mater Res. 20(2): 339348.

[18] A. F. Ismail, L. I. B. David. 2001. A Review on the Latest Development of Carbon Membranes for Gas Separation. $J$. Membr. Sci. 193: 1-18.

[19] Zhaodong Ding, Xuejiao Liu, Yang Liu and Liping Zhang. 2016. Enhancing the Compatibility, Hydrophilicity and Mechanical Properties of Polysulfone Ultrafiltration Membranes with Lignocellulose Nanofibrils. Polym. 8(349): 1-18.

[20] Hond Bin Li, Wen Ying Shi, Yu Feng Zhang, Dong Qing Liue and Xiao Feng Liu. 2014. Effects of Additives on the Morphology and Performance of PPTA/PVDF in Situ Blend UF Membranes. Polym. 6: 1846-1861.

[21] F. P. Cuperus and C. A. Smolders. 1991. Characterization of UF Membranes-Membrane
Characteristics

and

Characterization Techniques. Adv. Coll. Inter. Sci. 34: 135-173.

[22] G. Capannelli, F. Vigo and S. Munari. 1983. Ultrafiltration Membranes- Characterization Methods. J. Membr. Sci. 15: 289313.

[23] Izzati Izni Yusoff, Rosiah Rohani, Abdul Wahab Mohammad. 2017.

Molecular Weight Cut-off Determination of Pressure Filtration Membranes via Colorimetric Detection Method. Malaysian J. Anal. Sci. 21(2): 484-495.

[24] Venkatesh D. Vandekar. 2015. Manufacturing of Hollow Fiber Membrane. Int. J. Sci. Res. 23197064.

[25] Veeran Gowda Kadajji and Guru V. Betageri. 2011. Water Soluble Polymers for Pharmaceutical Applications. Polym. 3: 19722009.

[26] Jayant S. Phale and Yogesh J. Chendake. 2016. Polysulfone based Ultrafiltration Membrane Preparation by Phase Inversion: Parameter Optimization. Int. J. Sci. Res. 2319-7064.

[27] Xue Mei Tan and Denis Rodrigue. 2019. A Review on Porous Polymeric Membrane Preparation. Part I: Production Techniques with Polysulfone and Poly (Vinylidene Fluoride). Polym. 11(1160): 1-39.

[28] N. Misdan, W. J. Laua, A. F. Ismail, T. Matsuura. 2013. Formation of Thin Film Composite Nanofiltration Membrane: Effect of Polysulfone Substrate Characteristics. Desalination. 329: 9-18.

[29] G. Arthanareeswaran, V. Starov. 2010, Effect Of Solvents on Performance of Polyethersulfone Ultrafiltration Membranes: Investigation of Metal Ion 
Separations.

Desalination. 267(1): 57-63.

[30] Helen Julian and I. G. Wenten. 2012. Polysulfone Membranes for $\mathrm{CO} 2 / \mathrm{CH} 4$ Separation: State of the Art. IOSR J. Eng. 2(3): 484-495.

[31] Maria Tomaszewska and Anna Jarosiewicz. 2002. Use of Polysulfone in Controlled-release Npk Fertilizer Formulations. $J$. Agric. Food Chem. 50: 46344639.

[32] Divya Nagaraju, Deepti G. Bhagat, Rahul Banerjee and Ulhas K. Kharul. 2013. In-situ Growth of Metal Organic Frameworks on Porous Ultrafiltration Membrane for Separation Applications. $J$. Mater. Chem. 8828-8835.

[33] Xiangbao Liu, Huan Liu and Pei Li. 2017. Effect of Polymer Dope Concentration on the Morphology and Performance of PES/PDMS Hollow Fiber Composite Membrane for Gas Separation. Juniper Onl. J. Mater. Sci. 1(5): 1-5.

[34] Muhammad Usama Siddiqui, Abul Fazal Muhammad Arif and Salem Bashmal. 2016. Permeability-Selectivity Analysis of Microfiltration and Ultrafiltration Membranes: Effect of Pore Size and Shape Distribution and Membrane Stretching. Membr. 6(40): 1-14.

[35] Zhen-Liang $\mathrm{Xu}$ and F. Alsalhy Qusay. 2004. Polyethersulfone (PES) Hollow Fiber ultrafiltration Membranes Prepared by PES/nonsolvent/NMP Solution. J. Membr. Sci. 233: 101-111.

[36] V. Nassehi. 2011. Numerical Analyses of Bubble Point Tests Used For Membrane Characterization: Model Development and Experimental
Validation. Asia-Pac J. Chem. Eng. 6(6): 850-862.

[37] Hong-Bin Li, Wen-Ying Shi, Yu-Feng Zhang, Dong-Qing Liu and Xiao-Feng Liu. 2014. Effects of Additives on the Morphology and Performance of PPTA/PVDF in Situ Blend UF Membrane. Polym. 6: 1846-1861.

[38] Alumni Idris, Zakaria Man, Abdulhalim S. Maulud and Muhammad Saad Khan. 2017. Effects of Phase Separation Behavior on Morphology and Performance of Polycarbonate Membranes. Membr. 7(21): 1-18.

[39] G. B. Van Den Berg and C. A. Smolders. 1990. Flux Decline in Ultrafiltration Processes. Desalination. 77: 101-133.

[40] Tiziana Marino, Francesca Russo and Alberto Figoli. 2018. The Formation of Polyvinylidene Fluoride Membranes with Tailored Properties via Vapour/Non-Solvent Induced Phase Separation. Membr. 8(71): 1-17.

[41] Sagar Roy and Nayan Ranjan Singh. 2017. Polymeric Nanocomposite Membranes for Next Generation Pervaporation Process: Strategies, Challenges and Future Prospects. Membr. 7(53): 1-64.

[42] Guido Kickelbick. 2003. Concepts for the Incorporation of Inorganic Building Blocks into Organic Polymers on a Nanoscale. Prog. Polym. Sci. 28: 83-114.

[43] Rajesh Kumar Saini. 2012. Designing of Macroporous Polymeric Materials for Bioengineering Applications. Mater. Sci.: Indian J. 8(10): 389405.

[44] Beth A. Miller-Chou and Jack L. Koenig. 2003. A Review of Polymer Dissolution. Prog. 
74 Supriya S. Dhume, Pallavi Mahajan-Tatpate \& Yogesh J. Chendake

Polym. Sci. 28: 1223-1270.

[45] Sachin Mane. 2016. Effect of Porogens (Type and Amount) on Polymer Porosity: A Review. Canadian Chem. T. 4(2): 210225.

[46] Ulhas Kanhaiyalal Kharul, Ramchandra Vitthal Gadre,
Vithal Venkatrao Jogdand, Yogesh Jayasing Chendake. 2012. Polybenzimidazole Based Membrane for Deacidification. United States Patent. US 2012/0000852 A1. 5 January 2012. 